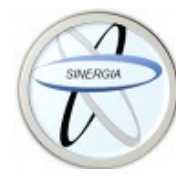

Revista Médica Sinergia

Vol. 6, Núm. 7, julio 2021, e688

\title{
Lesión pulmonar aguda relacionada a transfusiones sanguíneas
}

Transfusion - related acute lung injury

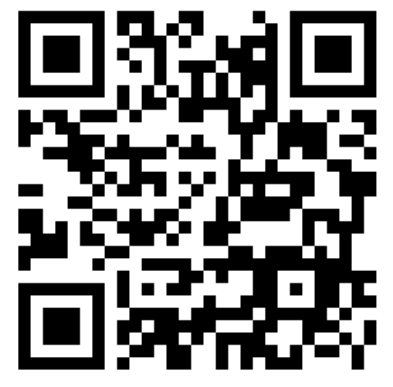

Recibido 27/04/2021
${ }^{1}$ Dra. Diana Melissa Rojas Sancho Investigadora independiente, Heredia, Costa Rica

(iD) https://orcid.org/0000-0001-8339-3134

${ }^{2}$ Dra. Laura Vanessa Bermúdez Durán Investigadora independiente, Limón, Costa Rica

(iD) https://orcid.org/0000-0002-4392-4794

${ }^{3}$ Dr. Mario Andrés Chacón Segura Investigador independiente, Alajuela, Costa Rica (i) https://orcid.org/0000-0003-0859-2517
Aceptado

20/05/2021

\section{RESUMEN}

La lesión pulmonar aguda producida por transfusión (TRALI) es un síndrome grave y potencialmente mortal. La FDA ha informado que TRALI es la principal causa muertes relacionadas con transfusiones durante muchos años, con el 34\% de las víctimas mortales de TRALI (64/186 reportaron muertes relacionadas con transfusiones entre 2012 y 2016). La asociación entre el antígeno humano de neutrófilos (HNA) o antígeno leucocitario humano (HLA) que se encuentran en los anticuerpos en sangre de donantes asocia los síndromes de TRALI y TACO. Con síntomas sugestivos dentro de las 6 horas posteriores a una transfusión sanguínea e infiltrados en una radiografía de tórax indicando de la presencia de edema pulmonar. Este síndrome ha mejorado mucho durante las dos últimas décadas, se han implementado medidas para mitigar el riesgo de TRALI en todo el mundo.

PALABRAS CLAVE: transfusión; anticuerpos; edema; hipoxemia.

\section{ABSTRACT}

Transfusion Acute Lung Injury (TRALI) is a serious and life-threatening syndrome. The FDA has reported that TRALI is the leading cause of transfusion-related deaths for many years, with $34 \%$ of TRALI fatalities (64/186 reported transfusion-related deaths between 2012 and 2016). The association between human neutrophil antigen (HNA) or human leukocyte antigen (HLA) found in antibodies in donor blood is associate to TRALI and TACO syndromes. With suggestive symptoms within 6 hours after a blood transfusion and infiltrates on a chest radiograph 
indicating the presence of pulmonary edema. This syndrome has improved a lot over the last two decades, measures to mitigate the risk of TRALI have been implemented around the world. KEYWORDS: transfusion; antibodies; edema; hypoxemia.

\footnotetext{
${ }^{1}$ Médica general, graduada de la Universidad de Ciencias Médicas (UCIMED). Cód. MED16075. Correo: dianarojas2495@gmail.com

2 Médica general, graduada de la Universidad de Ciencias Médicas (UCIMED). Cód. MED16050. Correo: bermudezlau96@outlook.es

${ }^{3}$ Médico general, graduado de la Universidad de Ciencias Médicas (UCIMED). Cód. MED16091. Correo: marioa0894@gmail.com
}

\section{INTRODUCCIÓN}

El término TRALI (debido a sus siglas en inglés "transfusión related acute lung injury") fue acuñado en 1983 (1,2), a la lesión pulmonar aguda asociada a la transfusión, sugerido por primera vez por el autor Popovsky et al. El primer caso reportado fue en la década de 1950 (3).

En 1966, Phillips et al informo sobre tres pacientes transfundidos con presencia de edema pulmonar sin sobrecarga de líquidos. En 1970, Ward observó la aparición de edema pulmonar no cardiogénico posiblemente debido a anticuerpos antileucocitos y en 1971, Thompson publicó un caso sobre una reacción pulmonar al transfundir sangre no compatible. Pero no fue hasta 1983 cuando el término "TRALI" fue oficialmente reconocido como una entidad que produce la enfermedad, ya que se asoció debido a la transferencia pasiva de anticuerpos antileucocitos (3).

Este síndrome se produce a la colocación de productos hemáticos, el cual se presenta con síntomas como hipoxemia aguda y edema pulmonar no cardiogénico, con un rango de hasta 6 horas posteriores a la recepción de una transfusión $(1,2)$. Los componentes como plasma, plaquetas, crioprecipitado, granulocitos, glóbulos rojos, inmunoglobulinas y células madre $(2,3)$ son los de mayor relevancia.

Coincidiendo con la introducción la transfusión sanguínea genero un impacto en los riesgos asociados, siendo más común las infecciones transmitidas y la incompatibilidad. Este impacto disminuyó considerablemente por la revisión constante y el proceso de calidad de los últimos años (3). La morbilidad y la mortalidad por la transfusión genero un diagnóstico clínico, así como el Síndrome Distress Respiratorio del adulto (SDRA), la transfusión asociada a sobrecarga circulatoria (TACO) y TRALI tipo I (sin factor de riesgo SDRA) y tipo II (con un riesgo de SDRA) $(1,2)$.

Este artículo tiene como objetivo el integrar la información necesaria, con el fin de exponer una adecuada compresión y descripción de los aspectos más relevantes para un diagnóstico precoz. Ya al ser una patología que requiere una alta sospecha clínica, se pretende enfocar hacia su patogenia, signos y síntomas para dar un abordaje oportuno y conseguir un pronóstico más favorable al paciente.

\section{MÉTODO}

El articulo presentado a continuación es una revisión bibliográfica, en donde se incluyen publicaciones con máximo de 5 años previos a la fecha. Se extraen de bases de datos como Pubmed, Medline, Elsevier, Uptodate y buscadores como Google Académico. Durante la revisión se eligen los que presentan en su contenido mayor relevancia clínica y académica. Se utilizó un total de 15 referencias bibliográficas.

\section{DEFINICIÓN}

El Consenso Canadiense define los criterios de TRALI como enfermedad pulmonar aguda y al edema después de la transfusión en ausencia de sobrecarga circulatoria o factores de riesgo del SDRA $(1,4)$. Por el 
contrario, TACO es el edema pulmonar principalmente relacionado con la sobrecarga circulatoria (5). El consenso sobre los criterios diagnósticos ha obstaculizado la clínica, el conocimiento e investigación de las reacciones a las transfusiones pulmonares.

La nueva definición de TRALI se define como TRALI tipo I (pacientes que no tienen factores de riesgo de SDRA) y el tipo II es en pacientes que tienen factores de riesgo de SDRA (6).

\section{INCIDENCIA}

Al ser un diagnóstico clínico demostró que aun esta patología se encuentra en subdiagnóstico. La incidencia de TRALI en norteamérica (incluido Canadá) ha oscilado de $1 / 100.000-1 / 10.000$ transfusiones (depende del producto hemático transfundido) y de 1/5.000-1/1.323 transfusiones en EE. UU. En Europa ha sido más infrecuentemente, con incidencias que oscilan entre 1,3/1.000.000-1/7.900 (5).

La incidencia real del síndrome no es conocida ya que históricamente, las estimaciones de incidencia de TACO y TRALI se han basado en la notificación de eventos adversos de los sistemas nacionales de hemovigilancia.

Todos los componentes sanguíneos se han implicado, pero tiene un aumento considerable de riesgo en aquellos que contienen plasma.

En el caso de las plaquetas la incidencia depende de su forma de obtención, por lo que ésta se estima aproximadamente un caso por cada 432 unidades cuando las plaquetas se obtienen de sangre total, y en un caso por cada 1.224 unidades cuando se obtienen mediante aféresis (6).

Las tasas de incidencia notificadas de TACO y TRALI han variado según la población de pacientes estudiada o el tipo de componente (7).

\section{FISIOPATOLOGÍA}

La primera evidencia que apoya el papel de los aloanticuerpos en la patogenia de TRALI que se publicó en 1983 (2). Se encontró que los pacientes con TRALI tenían leucoaglutinantes y anticuerpos linfocitotóxicos en el suero del hemoderivado transfundido.

Investigadores documentaron la asociación entre TRALI y antígeno humano de neutrófilos (HNA) o antígeno leucocitario humano (HLA) que se encuentran en los anticuerpos en sangre de donantes. Las investigaciones en laboratorio pueden incluir la determinación de recuentos de glóbulos blancos (leucopenia transitoria y trombocitopenia leve en TRALI), la detección de anticuerpos contra el receptor HLA clase I / II o antígenos HNA en el donante de sangre (alrededor del $80 \%$ de los casos de TRALI contienen estos hallazgos en sangre periférica). Sin embargo, se desconoce si los recuentos de células sanguíneas se pueden ver afectados y si los antileucocitos los anticuerpos podrían estar presentes (3). Por lo que se crean dos modelos:

- Modelo de "dos golpes" ("two hits"): este surge de una hipótesis de "dos resultados" que afirma que TRALI, es el resultado de dos impactos clínicos:

- El primer impacto es el resultado de la condición clínica del paciente, implica el secuestro de neutrófilos y que causa la activación del endotelio pulmonar. Esto conduce al secuestro de leucocitos polimorfonucleares (PMN) al endotelio pulmonar activado. Estos PMN activados son responsables de la liberación de citosinas y expresión de moléculas de adhesión (4). 
- El segundo impacto es mediado por anticuerpos en la transfusión de plasma que contiene anticuerpos específicos dirigidos contra antígenos en la superficie de PMN. Los anticuerpos antileucocitos del donante interactúan con el receptor neutrófilos, monocitos y endotelio pulmonar. Esto a su vez activa estos PMN, lo que resulta en la liberación de proteasas que inducen a fuga capilar. La acumulación de mediadores proinflamatorios y lípidos bioactivos de la sangre transfundida provoca edema pulmonar $(1,4)$ En aproximadamente el $80 \%$ de los casos, antígeno leucocitario antihumano (HLA) de clase I o II o los anticuerpos del antígeno de neutrófilos (HNA) están implicados en la activación de TRALI (3).

- Modelo de umbral: esta hipótesis no se ajusta a TRALI, se ha propuesto el modelo de umbral para explicar los pacientes que no son de alto riesgo y sin predisposición. Este modelo toma en cuenta la predisposición o lesión relativa del paciente, así como la fuerza de uno o más mediadores, incluidos anticuerpos, por tanto, asume que los pacientes pueden desarrollar siendo en un individuo sano $(1,4)$.

- Perfiles de citosinas: las proinflamatorias como IL-6 significativamente elevado en los pacientes y IL-8 no estaban elevados en pacientes con TACO después de la transfusión, comparado en los pacientes con TRALI, los niveles de IL-6 e IL-8 fueron elevado antes y después de la transfusión. Además, se encontró que la citosina antiinflamatoria IL-10 estaba aumentada tanto antes como después de la transfusión en TRALI. Los niveles bajos de IL-8 en combinación con los niveles elevados de IL-10 pueden potencialmente ayudar a respaldar un diagnóstico de TACO mientras que el aumento de los niveles de IL-8 en combinación con los niveles bajos de IL10 pueden ayudar potencialmente en el diagnóstico de TRALI $(1,3)$.

Al no haber evidencia directa de un papel prominente del endotelio en el desarrollo de TRALI, muchos parámetros giran en torno a él. Es de suma importancia para investigar el impacto de la vasculatura durante el inicio de TRALI. Los conocimientos se han obtenido de otros estudios sobre el papel del endotelio en cuanto a la inflamación. Las tasas de TRALI son más altas en pacientes que reciben una transfusión de plaquetas o plasma y aumenta a medida que aumenta el número de unidades transfundidas (4).

\section{DIAGNÓSTICO}

El diagnóstico preciso de estas reacciones se basa en la interpretación de la clínica, datos radiográficos y hemodinámicos. Los síndromes TACO y TRALI pueden ser difíciles de distinguir porque tienen un cuadro clínico similar. Hay estudios de ambos donde utilizan conocimientos de médicos, tanto en cuidados intensivos como en transfusiones sanguíneas en otras áreas de los hospitales $(7,8)$. Las reacciones transfusionales dan como resultado el edema pulmonar agudo dentro de las 6 horas posteriores a la transfusión; caracterizado por opacidades pulmonares bilaterales en la radiografía de tórax e hipoxemia por gasometría arterial $u$ oximetría de pulso. Dando un $\mathrm{PaO} 2$ / FiO2, 300 , SatO2 del $90 \%$ en el aire ambiente u otra evidencia de hipoxia $(1,7)$. La nueva definición de TRALI se define como TRALI tipo I y II (9): 
En el Tipo I se valoran pacientes que no tienen factores de riesgo de SDRA $y$ cumplen con los siguientes:

1. Inicio súbito o agudo

1.1. Hipoxemia $\mathrm{PaO} 2 / \mathrm{FiO} 2 \leq 3000$ SatO2 $<90 \%$ en el aire ambiente.

1.2. Evidencia de edema pulmonar bilateral por imágenes (como, por ejemplo, radiografía de tórax, TAC tórax o ultrasonido).

1.3. No hay evidencia de hipertensión auricular izquierda que se relacione con sobrecarga de volumen.

2. Inicio durante o dentro de las primeras seis horas posteriores al inicio de la transfusión sanguínea.

3. No hay relación temporal con un factor de riesgo para SDRA.

Por el contrario, el tipo II es en pacientes que tienen factores de riesgo de SDRA (pero que no han sido diagnosticados) o que tienen enfermedad preexistente (PaO2/FiO2 de 200-300), en donde el patrón respiratorio se deteriora posterior al proceso de la transfusión $(9,10)$. En los hallazgos descritos en las categorías a y b de TRALI tipo I, el estado respiratorio se encuentra estable en las 12 horas previas a la transfusión; es decir la aparición de síntomas pulmonares debe estar dentro de las 6 y hasta 72 horas posteriores a la transfusión $(3,9)$.

Se ha propuesto una modificación de la nomenclatura con respecto al TRALI con el fin de distinguir los casos de TRALI como "mediado por anticuerpos", "no mediado por anticuerpos" o "sin clasificación adicional posible", este último en los casos es en donde no se realiza ninguna investigación inmunológica. Este enfoque pretende eliminar el término "posible TRALI", cambiando el nombre de esta categoría como "SDRA transfundido" (6).

\section{PRESENTACIÓN CLÍNICA}

Es un síndrome que establece a la presentación súbita de dificultades respiratorias durante o después de una transfusión sanguínea. Dentro de sus signos podemos denotar hipoxemia e infiltraciones pulmonares en radiografías de tórax en la mayoría de los pacientes y la mitad de los pacientes muestran un esputo rosado $y$ espumoso (hemoptisis) $(4,5)$.

Entre otros se encuentra la taquipnea, la taquicardia, la presión elevada en las vías respiratorias, la fiebre, la hipotensión y la cianosis periférica en menos cantidad. Ninguna prueba de laboratorio es específica para diagnosticar. El mayor cambio es una caída transitoria en el recuento periférico de neutrófilos (posiblemente al secuestro de neutrófilos en la vasculatura pulmonar). Leucopenia se produce en el $35 \%$ de los pacientes después de la transfusión con un producto sanguíneo que contiene anticuerpos $(8,11)$.

TRALI debe distinguirse del edema pulmonar debido a otras causas con las que se comparten signos y síntomas. Como lo son las reacciones a transfusiones hemolíticas y sépticas ya que la clínica suele ser similar. La anafilaxia puede causar insuficiencia similar a la de TRALI, pero las señales de las vías respiratorias y síntomas son más comunes en pacientes con anafilaxia. Por otro lado, TACO es otra causa de insuficiencia respiratoria relacionada con transfusiones. Pero este posee un deterioro de la función miocárdica, rápida y agresiva a la terapia con fluidos se sugieren factores de riesgo para TACO $(6,7)$.

Lo que logra distinguir a TRALI es que tiene más probabilidad de estar asociado con signos y síntomas de inflamación, incluyendo fiebre, hipotensión y un infiltrado. Por lo que es más probable que TACO se asocie con hallazgos sugestivos de 
disfunción cardíaca y sobrecarga de volumen. Además, que incluye tres o más de los siguientes dentro de las 6 horas posteriores a la transfusión: dificultad respiratoria aguda, radiográfico: edema pulmonar, evidencia de insuficiencia cardíaca dado por el péptido natriurético de tipo $B$ elevado (BNP) y un resultado positivo del equilibrio de fluidos $(5,6)$. TACO se caracteriza por ser edema pulmonar cardiogénico, mientras que TRALI se presenta como edema pulmonar por aumento de la permeabilidad (no cardiogénico).

\section{FACTORES DE RIESGO}

La enfermedad crítica es uno de los mayores riesgos de TRALI, hay muchas comorbilidades como factores de riesgo estos incluyen (5):

- Enfermedad hepática en etapa terminal

- Derivación de arterias coronarias

- Neoplasias hematológicas

- Transfusión masiva

- Ventilación mecánica

- Sepsis

- Consumo elevado de alcohol

Además, aumento el impacto en las mujeres caucásicas mayores de 65 a 79 años con una historia de 6 meses de enfermedad pulmonar post inflamatoria como la fibrosis y el consumo de tabaco tienen una mayor probabilidad de desarrollar TRALI. La hemorragia posparto no se ha asociado con TRALI en la literatura $(1,4)$.

\section{PRONÓSTICO}

La mortalidad es del 5-10\%, sin embargo, la mortalidad a 90 días asociada con TRALI puede alcanzar $47 \%$ en poblaciones críticamente enfermas (6). Los casos sospechosos de TRALI deben notificarse de inmediato a el banco de sangre, en donde debe investigarse todos los donantes para la presencia de anticuerpos anti-HLA y antiHNA para identificar donantes con estos anticuerpos y evitar que donación futura (4). No existe una pauta a seguir con respecto a la transfusión de los pacientes. El personal médico debe considerar muchos factores de riesgo, socioeconómicos, en beneficio al paciente al decidir transfundir pacientes. Cuando se valoran pacientes anémicos, en lugar de basar la decisión únicamente en un nivel de laboratorio específico se debe considerar el impacto de este procedimiento. La decisión final de transfundir debe incorporar la condición clínica del paciente, comorbilidades y los deseos individuales del paciente.

Por otro lado, la utilización de la investigación sobre el mecanismo de activación endotelial. Como el endotelio parece estar involucrado como actor clave en todas las formas de TRALI mediado por anticuerpos que bloquea la activación del endotelio en la aparición de este síndrome puede ser el inicio para prevenir este síndrome potencialmente mortal (8).

La mortalidad se ha estimado en el $1-10 \%$ y según los datos de la Food and Drug Administration (FDA) y fue la causa más frecuente de muerte relacionada con la transfusión sanguínea en EE. UU. entre 2001 y 2003 (3).

\section{ESFUERZOS PARA MITIGAR EL TRALI}

Las estrategias de mitigación de TRALI han logrado reducir significativamente la incidencia. Hubo una reducción en los casos inducidos por plasma en poblaciones de riesgo y una tendencia a la disminución de los casos inducidos por plasma en un paciente general. La FDA informó de 35 casos de TRALI en 2006 y sobre la introducción de la mitigación se redujo a 8 casos en 2016 (3). Dos medidas para mitigar 
el TRALI se implementaron en el 2010: primero, el nuevo plasma congelado (PFC) y los concentrados de plaquetas de aféresis (APC) se restringieron los siguientes donantes: hombres, mujeres nulíparas o mujeres con hijos que fueron negativos para HLA anticuerpos de clase I y II $(1,6)$. El segundo las donaciones de sangre total de mujeres con HLA conocido los anticuerpos no se utilizaron para preparar concentrados de plaquetas de capa leucocitaria agrupados (BCPC).

El impacto de la solución de aditivo plaquetario (Platelet Additive Solution) después de la observación de que el uso, estaba relacionado con una reducción dramática de las reacciones alérgicas en los pacientes (6). Además, no se observó ningún efecto significativo sobre la mortalidad por TRALI a 30 días con pacientes de bajo riesgo.

En la exclusión de donantes femeninas ya que casi el $90 \%$ de los casos, ya sea anticuerpos HLA o HNA se detectaron en el plasma de los donantes. La implementación de un donante predominantemente masculino en las pautas de transfusión de PFC impacto en una disminución en la incidencia en el Reino Unido seguido de otros países del mundo. En un estudio retrospectivo de casos y controles realizado por Welsby et al (1) comparando pacientes que se sometieron a cirugía y recibieron plasma de donante masculino o femenino, encontraron resultados contrarios los receptores de plasma de donantes femeninas tenía menos disfunción pulmonar, menos muerte y hospitalización $(3,5,6)$.

\section{TRATAMIENTO}

Desafortunadamente, tanto para TACO como para TRALI, solo se dispone de medidas de soporte y no hay terapias específicas. Las medidas de soporte para TACO pueden incluir diuresis, oxígeno, e intubación. Para TRALI, las medidas de soporte pueden incluir oxígeno, intubación, manejo de soluciones intravenosas y vasopresores para mantener la hemodinamia (10).

Al referirnos al oxígeno suplementario, esto incluye el ventilador no invasivo y soporte del ventilador según sea la necesidad del paciente y la gravedad de su condición. Se han descrito grados de severidad (6), los cuales definen la base de la terapia requerida:

- Grado 1 (leve): presenta recuperación espontánea al suministrar el oxígeno suplementario a través de una mascarilla.

- Grado 2 (grave): estos casos requieran ventilación no invasiva o traslado a la unidad de cuidados intensivos por monitorización constante.

- Grado 3 (potencialmente mortal): casos que requieren ventilación mecánica invasiva con o sin terapia adicional como el uso de vasopresores según lo requieran sus signos vitales.

- Grado 4 (muerte): casos en los que existe una clara relación temporal entre la muerte y TRALI.

Se han utilizado de forma anecdótica en los informes de casos de TRALI, pero no existen estudios a gran escala (11). La utilización de la investigación sobre el mecanismo de activación endotelial, como el endotelio parece estar involucrado como actor clave en todas las formas de TRALI. Esto mediado por anticuerpos que bloquean la activación del endotelio en la aparición puede ser preventivo este síndrome potencialmente mortal (12).

Con respecto a otras terapias utilizadas se encuentran los corticoesteroides, aunque pueden ser efectivos para disminuir la mortalidad y tiempo para lograr la respiración sin ayuda en SDRA y en unidad de cuidados intensivos. 
hay datos limitados que demuestren la eficacia (13). Demostraron disminuciones en los marcadores de inflamación sistémica, los estudios en animales sugieren que la metilprednisona puede disminuir los niveles de IL-6 (sugestivo de inflamación sistémica), pero no edema pulmonar. Se menciona este uso a altas dosis, pero no cuenta con estudios clínicos que demuestren su eficacia $(10,14)$.

En cuanto a los agentes antiplaquetarios hay modelos y estudios en pacientes humanos no concluyentes. El papel del tratamiento con aspirina prevenido en TRALI aparentemente al disminuir el secuestro de plaquetas en los pulmones, aunque no mostró un efecto protector (12).

Por otro lado, se requerirá más validación y confirmación para otras terapias centradas en el receptor de la transfusión o para intervenciones terapéuticas dirigidas al producto de transfusión de sangre. Las investigaciones con respecto a la terapia con IL-10 demostró no solo ser profiláctica sino también terapéuticamente para prevenir y rescatar TRALI. Dado que los niveles de IL10 no están elevados en los pacientes con TRALI postransfusión, IL-10 la terapia puede ser particularmente interesante de explorar como una terapia potencial $(11,13)$.

Dentro de los casos con efectos adversos a la transfusión, existe cierto cuestionamiento acerca de reiniciar la transfusión del producto original. Sin embargo, esta no debe continuarse en casos de sospecha de reacción transfusional hemolítica aguda (AHTR), anafilaxia, sepsis o TRALI (14). Deben realizarse pruebas de HLA y anticuerpos (anti-HLA), además se debe informar al centro de recolección de sangre de un caso sospechoso de TRALI para que se puedan obtener las muestras apropiadas del donante y la notificación correspondiente (15).

\section{CONCLUSIONES}

El consenso para TRALI trajo consigo una mejor comprensión de su patogenia. Está claro que el personal en salud debe prestar especial atención a los factores de riesgo del paciente e implementar practicas basadas en evidencia. Esto por consiguiente debe evitar transfusiones cuando no están indicadas. El enfoque al diagnóstico clínico, en su mayoría tardío y los síntomas más significativos son abordados con medidas de soporte para tener un pronóstico favorable ya que nos encontramos ante una patología que puede comprometer la vida causando la muerte al ser tiempo dependiente.

\section{Los autores declaran no tener conflicto de interés.}

\section{REFERENCIAS}

1. Semple J., Rebetz J., Kapur R. Transfusionassociated circulatory overload and transfusionrelated acute lung injury. Blood Journal.org (Internet). 2019; (1) 1-28, Disponible https://doi.org/10.1182/blood-2018-10-860809

2. Otrock K., Lui C., Grossman J. Transfusionrelated acute lung injury risk mitigation: an update. ISBT (Internet). 2017. Vox Sanguinis 1 a 10, Disponible en https://doi.org/10.1111/vox.12573

3. Semple J., Rebetz J., Kapur R. Transfusionassociated circulatory overload and transfusionrelated acute lung injury. Blood Journal.org (Internet). 2019; (1) 1-28, Disponible en https://doi.org/10.1182/blood-2018-10-860809

4. Morsing, K. S. H., Peters, A. L., van Buul, J. D., \& Vlaar, A. P. J. The role of endothelium in the onset of antibody-mediated TRALI. (Pubmed). 2018. Blood Reviews, 32(1), 1-7. Disponible en https://doi.org/10.1016/j.blre.2017.08.003

5. Rojas C. TRALI. Lesion pulmonary aguda relacionada con transfusion sanguínea. Revista Mexicana de Anestesia en Trauma (Internet). 2016. Vol. 39 (1) S48-S52. Disponible en https://www.medigraphic.org.mx

6. Andreu G., Boudjedir K., Muller J., et al. Analysis of TRALI and possible TRALI reported to the French Hemovigilance Network from 2007 to 2013. Transfusion Medicine Reviews (2017), Manuscripy (1-48). Disponible en https://doi.org/10.1016/j.tmrv.2017.07.001 
7. Roubinian N. TACO and TRALI: biology, risk factors, and prevention strategies. Blood Systems Research Institute, San Francisco, CA. American Society of Hematology 2018 (Internet). 2020. Update 586-594. Disponible en https://ashpublications.org/hematology/articlepdf/2018/1/585/1255520/hem01876

8. Taffarel P., Meregalli C., Baron F., et al. Lesión pulmonar aguda secundaria a transfusión (TRALI): reporte de un caso y revisión de la bibliografía. Medicina Intensiva Hospital de Niños de Buenos Aires (Internet). 2016. 33 (1). Disponible en https://www.revista.sati.org.ar

9. Bockhold C., Crumpler S. Reacciones transfusionales relacionadas con los pulmones. Nursing (2016) (Internet). TEK IMAGE/SCIENCE SOURCE. Volumen 33 (3) pp 37-41.

10. Kuldane S., Kelher M., Silliman C. Risk factors, management and prevention of transfusionrelated acute lung injury: a comprehensive update. Expert Review of Hematology (Internet). Accepted Jul 2019. Disponible en https://doi.org/10.1080/17474086.2019.1640599

11. De Clippel, D., Emonds, M.-P., \& Compernolle, V. Are we underestimating reverse TRALI? (2019) (Internet) Case Report.Transfusion 1- 6. https://doi:10.1111/trf.15431

12. Kleinman $S$, Kor D. Transfusion-related acute lung injury (TRALI). (2020) (Internet). Up to date. Review 2021. Wolters Kluwer Health, 230 Third Avenue

13. Waltham, MA 02451. Disponible en Transfusionrelated acute lung injury (TRALI) - UpToDate

14. Silvergleid A., Kleiman S, Tirnauer J. Approach to the patient with a suspected acute transfusion reaction. (2021) (Internet). Wolters Kluwer Health, 230 Third Avenue

15. Waltham, MA 02451. Disponible en Approach to the patient with a suspected acute transfusion reaction - UpToDate

16. Giverez M., Gottlieb S., Finlay G., Non cardiogenic pulmonary edema. (2019) (Internet). Wolters Kluwer Health, 230 Third Avenue

17. Waltham, MA 02451. Disponible en Noncardiogenic pulmonary edema - UpToDate

18. Siegel M., Parsons P., Finlay G. Acute respiratory distress syndrome: Clinical features, diagnosis, and complications in adults. (2020) (Internet). Wolters Kluwer Health, 230 Third Avenue Waltham, MA 02451. Disponible en Acute respiratory distress syndrome: Clinical features, diagnosis, and complications in adults - UpToDate 